\title{
Pseudophakodonesis and corneal endothelial contact: direct observations by high-speed cinematography
}

\author{
PAUL M. JACOBS, HUNG CHENG, AND NICHOLAS C. PRICE \\ From the Eye Hospital, Walton Street, Oxford, and the Nuffield Laboratory of Ophthalmology, \\ Walton Street, Oxford
}

SUMmARY High-speed cinematography was used to observe the movement of Federov type I lens implants within the anterior chamber. Our measurements suggest that in most patients contact between the lens implant and corneal endothelium does not occur.

A feature of iris clip intraocular lens implants following intracapsular cataract extraction is the considerable movement of the implant within the eye (pseudophakodonesis) that can follow even minor eye movements. It has been suggested that such movement may be sufficiently great for parts of the implanted lens to touch the corneal endothelium intermittently and thus produce continuing endothelial cell damage.

Jagger and $\mathrm{Jacobi}^{1}$ described an indirect method of analysing pseudophakodonesis by filming the movement of the light reflex from the front of the lens implant. We have already described and demonstrated a way of observing lens implant movement directly using high-speed cinematography. ${ }^{2}$

This paper presents the results of a quantitative study using this technique to assess the degree of movement of Federov type I iris clip lens implants following intracapsular extraction.

\section{Subjects and methods}

We examined 60 patients who had intracapsular cataract extraction with the implantation of a Federov type I lens implant performed between 2 and 24 months previously. The Federov type I lens is an iris clip lens implant similar to the Binkhorst implant. There is a $5.0 \mathrm{~mm}$ diameter Perspex lenticulus placed anterior to the iris and supported by 2 anterior and 2 posterior nylon loops. The anterior loops measure $7 \cdot 5$ $\mathrm{mm}$ from the apex of one loop to the apex of the other and, unlike the Binkhorst lens, are arranged at rightangles to the posterior loops. The implant is normally

Correspondence to Dr P. Jacobs, Moorfields Eye Hospital, City Road, London EC1V 2PD. inserted with the anterior loops placed horizontally across the anterior chamber. In our series the implant was stabilised either by suturing one anterior loop to the iris with a 10/0 monofilament suture or by use of a nylon peg attached to the lenticulus which engaged one posterior loop through an iridectomy.

Of the 60 patients 5 were selected who appeared to have very mobile implants and who were willing to participate in the study. All 5 patients were filmed initially, and on the basis of these preliminary findings 2 patients showing the greatest amplitude of implant movement were selected for further filming and quantitative assessment.

Patients were seated with the eye to be filmed immersed in a bath containing Ringer's solution. Illumination was provided by a fibreoptic light guide dipped into the Ringer's solution, and filming was from above with a fixed camera. Film speed was $\mathbf{2 0 0}$ frames per second, and Kodak Eastman 7247 colour negative film was used throughout. The eye was filmed under Ringer's solution in order to minimise corneal refraction, thus giving a true impression of the profile of the anterior chamber. ${ }^{2}$

The patients were instructed to perform voluntary horizontal eye movements as rapidly as they could. The eye position and movement were controlled by target lights fixed by the patient's other eye, which was not enclosed in the bath of Ringer's solution. Several sequences of movement were filmed for each patient. Fig. 1 illustrates a typical frame from such a sequence.

Analysis of individual frames was performed by tracing the image as illustrated in Fig. 2, so that the optic axis $O$ and the implant axis I could be determined. Because the camera was fixed, change of $O$ in relation to the edge of the frame is a measure of 


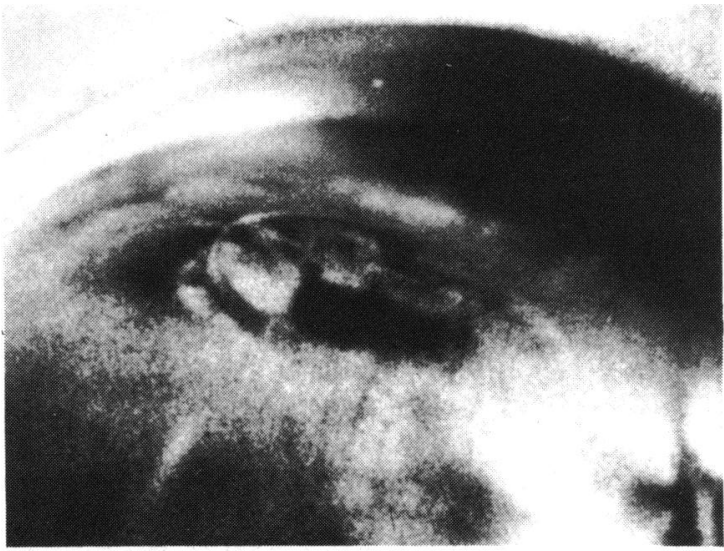

Fig. 1 Single frame taken from a $16 \mathrm{~mm}$ colour film sequence showing implant tilt of $13^{\circ}$.

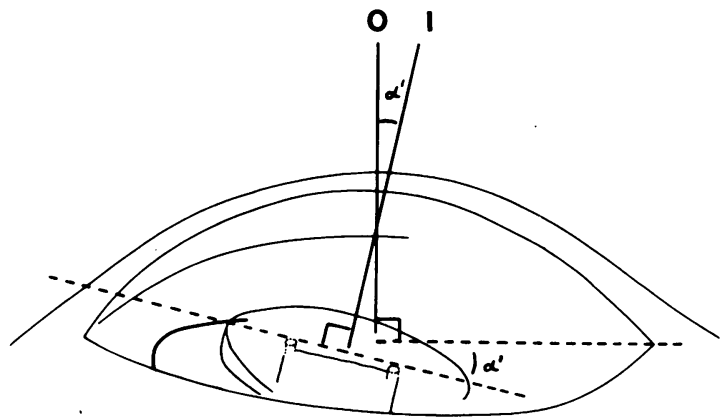

Fig. 2 Diagram of Fig. 1 to illustrate method of frame analysis.

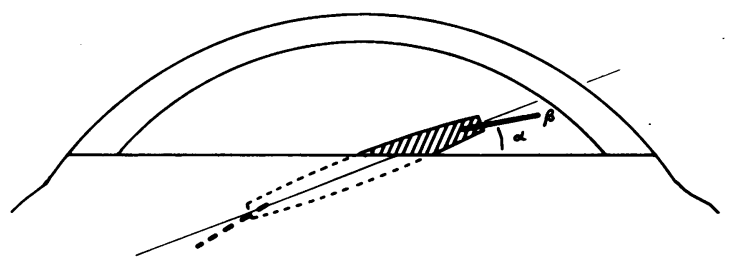

Fig. 3 Horizontal profile of anterior chamber showing tilt of lens implant $(\alpha)$ and distance of anterior loop from endothelium $(\beta)$.

eye movement. The difference between $O$ and $I$, the angle $\alpha^{\prime}$ is a measure of the tilt of the implant within the eye at that particular instant. Because of the slight upward tilt of the optic axis, the observed angle $\left(\alpha^{\prime}\right)$ is slightly smaller than the true horizontal angle $(\alpha)$ shown in Fig. 3. This difference can be allowed for by using the relationship:

$$
\tan \alpha=\frac{\tan \alpha^{\prime}}{\cos Y}
$$

where $Y$ is the upward tilt of the optic axis. The angle $Y$ for a sequence was known from the arrangement of the fixation target lights and could be confirmed for any frame by measuring the difference between the apparent vertical and horizontal corneal diameters.

By carrying out frame by frame analysis in this fashion, the movement of the optic axis $(0)$, together with associated implant tilt $(\alpha)$ could be recorded. A typical sequence is illustrated in Fig. 4. During the first 75 milliseconds the optic axis has moved from about $4^{\circ}$ to the left to about $7^{\circ}$ to the right (a total of $\left.11^{\circ}\right)$. This has induced an immediate tilt of the lens implant within the eye of $20^{\circ}$, with subsequent oscillations of diminishing magnitude until the implant comes to rest at about $\mathbf{2 3 0}$ milliseconds after the start of the sequence. The solid dots on the graph indicate individual frame measurements. This graph also shows the velocity of eye movement (the gradient of the dotted line is the angular velocity of the optic axis in degrees per millisecond). From this, by plotting velocity against time, it is possible to derive the angular acceleration of the optic axis.

In addition to the cinematographic observations, static measurements of anterior chamber depth and the distance of the anterior surface of the lens implant from the corneal endothelium were made with the Haag-Streit slit-lamp 900 with anterior chamber depth measuring attachment. Radial keratometry was performed with the Haag-Streit keratometer on all patients before and after surgery.

\section{Results}

Implant tilt. In none of the sequences that we filmed did the Federov type I lens implant appear to tilt sufficiently for any part of the implant to come near

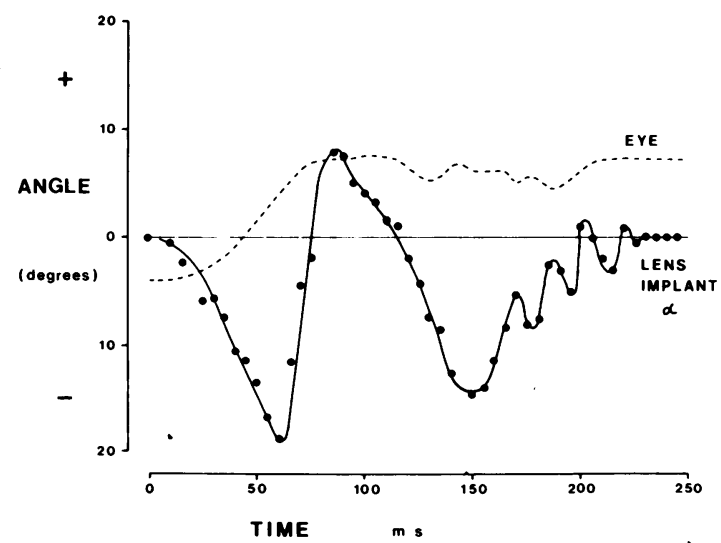

Fig. 4 Graph showing a single sequence of eye and lens implant movement obtained by the method of frame by frame analysis described in the text. The solid spots indicate individual measurements. 
the corneal endothelium. Frame by frame analysis showed the largest amplitude of tilt measured in any sequence to be $20^{\circ}$, which in that particular patient corresponded to a proximity of the loop to the endothelium of $1.9 \mathrm{~mm}$.

Relationship between angular acceleration of the optic axis and tilt of the lens implant. Eight separate sequences of eye and implant movement were analysed, 5 from patient 1 and 3 from patient 2 . Angular acceleration was estimated as described above. In Fig. 5 the maximum angular acceleration of the optic axis has been plotted against the maximum associated tilt of the lens implant. The implication of this is discussed below.

Relationship between anterior chamber depth before surgery and the implant position following

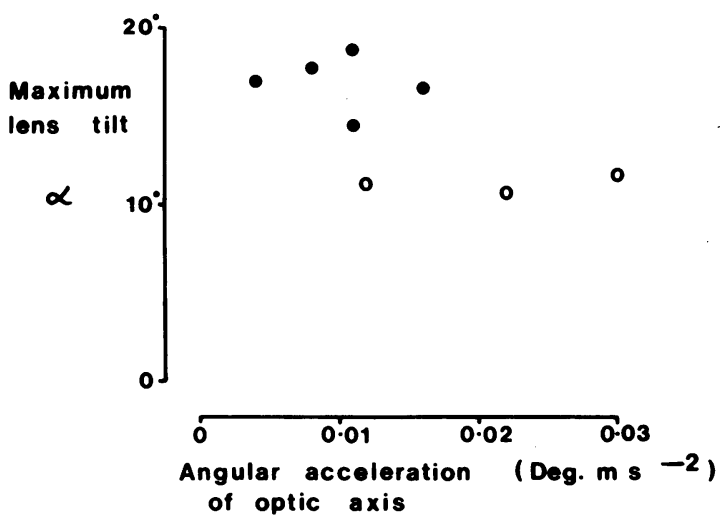

Fig. 5 The relationship between angular acceleration of the eye and tilt of the lens implant for 8 separate sequences of movement from 2 patients. The solid spots indicate patient 1 , the circles patient 2.

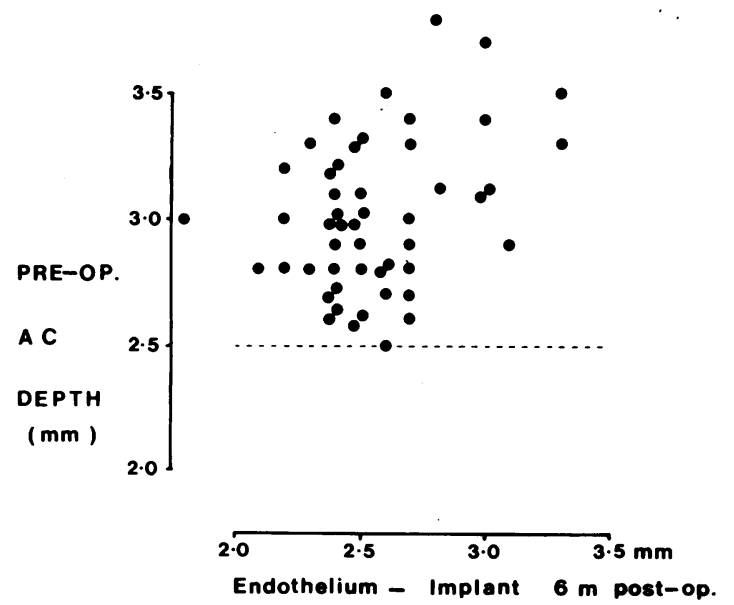

Fig. 6 Anterior chamber (A C) depth before surgery related to implant position 6 months (m) later. surgery. The anterior chamber depth, the distance from the anterior surface of the lens to the central corneal endothelium, was measured on the day prior to surgery for 50 consecutive cases. In Fig. 6 this is compared with the distance between the anterior surface of the lenticulus and the corneal endothelium at 6 months after surgery. Each point on the graph represents one patient. We did not perform this type of lens implantation in any patient with a preoperative anterior chamber depth of less than $2.5 \mathrm{~mm}$.

Effect of posture on the position of the Federov type I lens implant. The distance between the posterior surface of the cornea and the anterior surface of the implant is tabulated for 3 patients (Table 1 ) for 2 head positions-erect and prone.

Keratometry readings taken 6 months after surgery and expressed as the smallest radius of curvature are listed in Table 2. For 36 eyes the radius was $7.5 \mathrm{~mm}$ or greater, for 19 eyes the curvature was from $7 \cdot 0$ to $7 \cdot 4$ $\mathrm{mm}$, and for only 2 eyes was the value $6.9 \mathrm{~mm}$.

\section{Discussion}

Factors which affect the proximity of the iris clip implant to the cornea are the position of the implant at rest, tilt of the implant, decentering of the implant

Table 1 Distance between the anterior surface of the lenticulus and the central corneal endothelium (in millimetres) with change in head position

\begin{tabular}{llll}
\hline Patient & Vertical & Prone & \\
\cline { 3 - 4 } & & $5 \min$ & $10 \mathrm{~min}$ \\
\hline a & $2 \cdot 30$ & $2 \cdot 30$ & $2 \cdot 30$ \\
b & $2 \cdot 55$ & $2 \cdot 55$ & $2 \cdot 50$ \\
c & $2 \cdot 80$ & $2 \cdot 80$ & $2 \cdot 85$ \\
\hline
\end{tabular}

Table 2 Keratometry reading of 57 eyes 6 months after lens implant surgery

\begin{tabular}{lc}
\hline Minimum corneal radius $(\mathbf{m m})$ & Number of eyes \\
\hline $8 \cdot 2$ & 1 \\
$8 \cdot 1$ & 0 \\
$8 \cdot 0$ & 0 \\
$7 \cdot 9$ & 3 \\
$7 \cdot 8$ & 6 \\
$7 \cdot 7$ & 8 \\
$7 \cdot 6$ & 10 \\
$7 \cdot 5$ & 8 \\
$7 \cdot 4$ & 5 \\
$7 \cdot 3$ & 5 \\
$7 \cdot 2$ & 6 \\
$7 \cdot 1$ & 3 \\
$7 \cdot 0$ & 0 \\
$6 \cdot 9$ & 2 \\
Total & 57 \\
\hline
\end{tabular}


vertically or horizontally, and forward movement of the implant within the eye. For a given axial anterior chamber depth the implant will also be closer to the peripheral endothelium if corneal curvature is greater.

The maximum implant tilt (angle $\alpha$ ) that we observed as $20^{\circ}$ in one patient. All other patients that we filmed appeared to show smaller degrees of tilt than this. In our study filming was carried out with the patient's head fixed, and eye movement was by use of extraocular eye muscles only. In normal conditions much more vigorous movements may occur, for example, by shaking the head, participating in active sports, or riding in a motor vehicle. It is important to decide whether such violent eye movements, which we are unable to film, can induce a greater angle of tilt of the lens implant.

The results shown in Fig. 5 suggest that this is not the case. Although the 2 patients each show a different maximum implant tilt, about $20^{\circ}$ in patient 1 and $12^{\circ}$ in patient 2 , there is no increase in tilt with increased acceleration of the eye. Even the slower sequences that we analysed were sufficient to elicit the maximum implant tilt.

The further the implant is from the cornea when the eye is at rest, the greater it can tilt before it will touch the endothelium. The curve in Fig. 7 is derived theoretically on the assumption of a centrally situated Federov type I lens implant within a standard eye. The radius of curvature of the anterior surface of the cornea was taken as $7.8 \mathrm{~mm}$ and that of the posterior surface as $6.5 \mathrm{~mm}$. It can be seen that, if the distance between the anterior surface of the lenticulus and the central corneal endothelium is only $0.5 \mathrm{~mm}$, the anterior loops of the implant will touch the endothelium at rest. With increasing depth of anterior chamber the minimum tilt required increases, so that if the depth is more than $3.0 \mathrm{~mm}$ it is

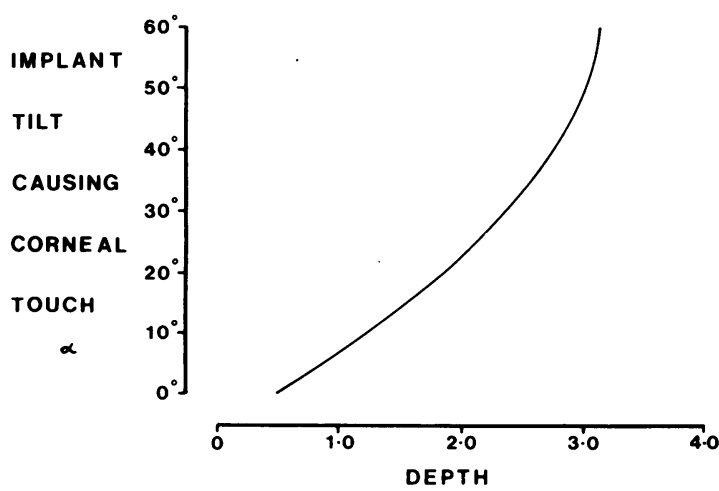

Fig. 7 Implant tilt ( $\alpha$ degrees) required for corneal contact according to the distance of the centre of the implant from the central corneal endothelium. almost impossible for endothelial contact to occur. The maximum tilt that we were able to measure in any patient was $20^{\circ}$. From Fig. 7 it can be seen that this will not result in corneal touch so long as the distance between the anterior surface of the lenticulus and the centre of the cornea is greater than $1.9 \mathrm{~mm}$.

We did not implant a Federov type I lens into any patient with a preoperative anterior chamber depth of less than $2.5 \mathrm{~mm}$. As illustrated in Fig. 6, of 50 consecutive cases only one patient had an anterior chamber depth of less than $1.9 \mathrm{~mm}$ at 6 months after surgery.

If the radius of curvature of the cornea is less than the $7.8 \mathrm{~mm}$ radius that we have assumed, then for a given depth of anterior chamber the loops will be closer to the peripheral endothelium. To estimate the importance of this factor we considered the minimum corneal radius, irrespective of axis, measured with the Haag-Streit keratometer at 6 months following surgery for 57 patients. In the great majority of our patients the minimum radius was not less than $7 \cdot 5$ $\mathrm{mm}$, but for 2 the value was $6.9 \mathrm{~mm}$. Even if we assume a radius of curvature of $6.9 \mathrm{~mm}$ for an eye, given the same set of assumptions regarding anterior chamber depth, centration, and corneal thickness, the tips of the anterior loops would only be $0.2 \mathrm{~mm}$ nearer the endothelium than they would be for an eye with a radius curvature of $7.8 \mathrm{~mm}$. We conclude, therefore, that in practice corneal curvature is not an important determinant of endothelial contact.

As illustrated in Fig. 8, movement of the implant away from the central position results in one anterior loop approaching the endothelium and the other moving away. Clearly if an implant is very eccentrically positioned, the danger of one anterior loop impinging on the corneal endothelium is increased. In the eyes that we filmed, however, side to side movement of the implant with movement of the eye was minimal.

Forward movement of iris clip lens implants within the eye with changes in posture does not appear to be important. Nordlohne ${ }^{3}$ measured the anterior chamber depth in both the supine and the prone

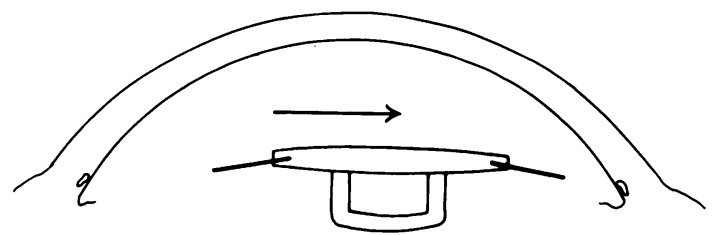

Fig. 8 Lateral movement of the implant within the anterior chamber causes one loop to approach the comeal endothelium so that less forward tilt is required for endothelial contact. 
position for 20 cases after intracapsular extraction with the implantation of a Binkhorst iris clip lens implant, a lens very similar to the Federov type I. He found a mean difference between supine and prone of only $0.24 \mathrm{~mm}$. We measured the difference between erect and prone for patients with Federov implants and found an even smaller change.

\section{CONCLUSION}

The method that we have described allows detailed observations of very rapid eye and lens implant movements. From our measurements it appears that pseudophakodonesis is not sufficient to cause a centrally situated Federov type I lens implant to touch the corneal endothelium unless the anterior chamber is shallow.

Although iris clip lenses are not the only type of lens available to the implant surgeon, many such implants continue to be used. If in such cases continuing endothelial cell loss is observed following surgery, an explanation other than direct contact between the lens implant and the cornea must be sought.

The cinematography for this study was performed by Mr I. G. Moar of Oxford Scientific Films, Long Hanborough, Oxon, England.

This study was supported in part by National Institutes of Health grant no. EYO 2677-03, the District Research Committee of the Oxfordshire Health Authority, Rayners Intraocular Lenses Ltd, and the Knoop Trust for Ophthalmic Research.

\section{References}

1 Jagger WS, Jacobi KW. An analysis of pseudophakodonesis and iridodonesis. J Am Intraocular Implant Soc 1979; 5: 203-6.

2 Cheng H, Jacobs $\mathrm{P}$, Price N, Moar I. Anterior chamber profile and lens implant movement-a high speed cinematographic study. Trans Ophthalmol Soc UK 1982; 102: 21-3.

3 Nordlohne ME. The intraocular implant lens: development and results with special reference to the Binkhorst lens. Baltimore: Williams and Wilkins, 1975: 231. 